\title{
Smoking out the masters: transcriptional regulators for nicotine biosynthesis in tobacco
}

\author{
Tsubasa Shoji*, Takashi Hashimoto \\ Graduate School of Biological Sciences, Nara Institute of Science and Technology, Ikoma, Nara 630-0192, Japan \\ *E-mail: t-shouji@bs.naist.jp Tel: +81-743-72-5524 Fax: +81-743-72-5529
}

Received January 9, 2013; accepted February 21, 2013 (Edited by T. Ariizumi)

\begin{abstract}
Nicotine and tropane alkaloids are specialized metabolites produced in certain species of Solanaceae, and some of these alkaloids have been used as pharmacological agents. In tobacco plants, nicotine is a defensive toxin against herbivorous insects, and jasmonate (JA) signaling leads to the induction of nicotine biosynthesis. JA-responsive structural genes of the nicotine pathway have been identified as being down-regulated in a low-nicotine tobacco mutant, which possesses mutant alleles at two loci, NICOTINE1 and NICOTINE2 (NIC1 and NIC2). A group of JA-responsive genes that encode homologous ERF transcription factors are clustered at the NIC2 locus and deleted in the mutant. These NIC2-locus ERFs up-regulate the structural genes of the biosynthetic pathway by recognizing GCC-like boxes in their promoters, forming a regulon for nicotine biosynthesis with the downstream targeted genes. The three basic components in JA signaling, COI1, JAZ, and MYC2, are required for JA-induced nicotine formation in tobacco. The bHLH transcription factor MYC2 positively regulates the structural genes, both directly by recognizing $\mathrm{G}$ boxes in their promoters and indirectly by up-regulating NIC2-locus ERF genes. Molecular elucidation of nicotine regulation would lead us to better understand the JA-dependent regulation of a wide range of phytochemicals.
\end{abstract}

Key words: nicotine, tobacco, ERF transcription factor, jasmonate signaling.

\section{Human use of Solanaceae alkaloids}

The family Solanaceae includes a handful of species that accumulate a group of highly toxic pyrrolidine alkaloids: tropane alkaloids in Atropa, Datura, and Hyoscyamus, and biogenetically related nicotine alkaloids in Nicotiana (Hashimoto and Yamada 1994) (Figure 1). While intoxication by these alkaloids is sporadically reported, their use by humans can be traced back to ancient times. The Egyptian queen Cleopatra (BC 69 to 30) is said to have used extract of Atropa belladonna, a representative medicinal plant which contains tropane alkaloids such as hyoscyamine and scopolamine, to dilate her pupils and so make herself more attractive as a political tactic. In the eighteenth century, a mixture including a tropanecontaining extract of Datura metel was used as a general anesthetic for the first time by the Japanese surgeon Seishu Hanaoka (1760 to 1835) to treat his wife's breast cancer. Atropine, a racemic mixture of two hyoscyamine forms, and scopolamine, are used as anticholinergic medicines that generally lower the parasympathetic activity of muscles and glands. Even today, due to their complex chemistry, these alkaloids are extracted from tissues of medicinal plants that are mainly cultivated in tropical plantations.

When Columbus explored the New World, its native inhabitants were already smoking the leaves of wild Nicotiana species indigenous to the region. Because of its addictive nature, smoking has become widespread around the world and tobacco (Nicotiana tabacum) is still one of the major crops cultivated worldwide (Goodman 1993). Of course, smoking is no longer recommended because of its markedly harmful effects on human health (Hecht 2003), and regulations on smoking are becoming increasingly restrictive nowadays. In tobacco products, nicotine is the major determinant of addiction to smoking, and reducing nicotine level is thus an important goal in tobacco breeding.

\section{Biosynthetic pathway}

Classical metabolic labeling experiments followed by enzymatic studies defined the biosynthetic pathways of the alkaloids (Figure 1; Hashimoto and Yamada 1994; Leete 1983). In particular, the use of cultured tissues and cells selected for high alkaloid production

Abbreviations: AO, aspartate oxidase; AP, APETALA; BBL, berberine bridge enzyme-like protein; bHLH, basic helix-loop-helix; COI, coronatine insensitive; ERF, ethylene response factor; JA, jasmonate; JAZ, jasmonate ZIM-domain; MATE, multidrug and toxic compound extrusion transporter; MPO, $N$-methylputrescine oxidase; NIC, NICOTINE; NUP, nicotine uptake permease; ODC, ornithine decarboxylase; PMT, putrescine Nmethyltransferase; QPT, quinolinate phosphoribosyltransferase; QS, quinolinate synthase.

This article can be found at http://www.jspcmb.jp/

Published online September 27, 2013 


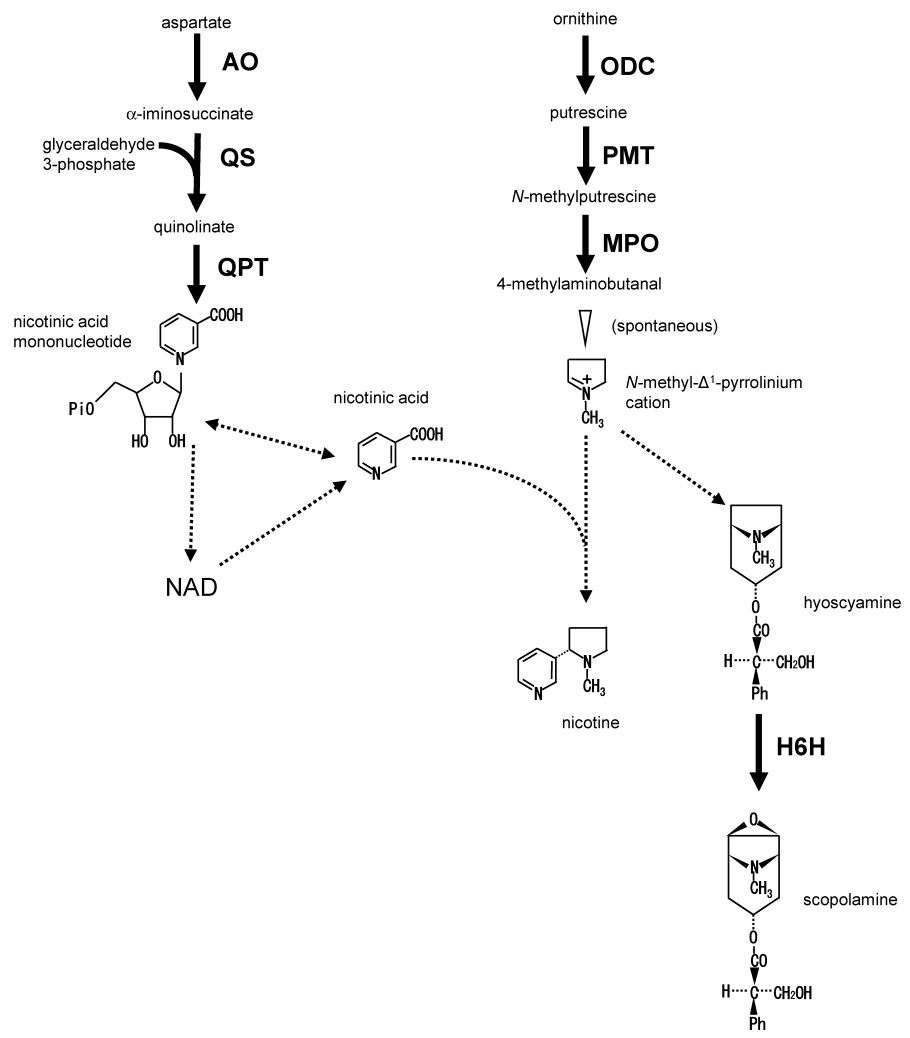

Figure 1. Biosynthesis pathways of nicotine and tropane alkaloids. Solid lines indicate defined steps, while broken lines indicate undefined steps or steps including multiple reactions. Abbreviations are as follows: ODC, ornithine decarboxylase; PMT, putrescine $N$-methyltransferase; MPO, $\mathrm{N}$-methyltransferase oxidase; $\mathrm{AO}$, aspartate oxidase; QS, quinolinate synthase; QPT, quinolinate phosphoribosyltransferase; $\mathrm{H} 6 \mathrm{H}$, hyoscyamine $6 \beta$-hydroxylase; $\mathrm{Pi}$, inorganic phosphate; $\mathrm{Ph}$, phenyl group.

facilitated detailed biochemical studies, such as the purification of enzymes that allowed the molecular cloning of corresponding genes. Efforts to clone alkaloid biosynthesis genes began in the early 1990s (Hibi et al. 1994; Matsuda et al. 1991) and became widespread with the advent of molecular biology (Facchini 2001; Hashimoto and Yamada 1994, 2003; Ziegler and Facchini 2008).

A pyrrolidine ring, which is incorporated into nicotine or further converted to a bi-cyclic tropane ring, is generated from ornithine through three consecutive reactions catalyzed by ornithine decarboxylase (ODC), putrescine $N$-methyltransferase (PMT), and $\mathrm{N}$-methylputrescine oxidase (MPO), and this part of the pathway is shared among all species producing pyrrolidine alkaloids (Hashimoto and Yamada 1994; Hibi et al. 1994; Shoji and Hashimoto 2011a; Shoji and Hashimoto 2013) (Figure 1). A pyridine ring, the other ring of nicotine, is derived from nicotinic acid, a primary metabolite in NAD metabolism, which occurs in every organism to supply the essential cofactor. In dicotyledonous plants, including tobacco, the pathway that generates NAD and nicotinic acid starts from aspartate, which is converted to nicotinic acid mononucleotide through reactions catalyzed by aspartate oxidase (AO), quinolinate synthase (QS), and quinolinate phosphoribosyltransferase (QPT) (Katoh and Hashimoto 2004; Katoh et al. 2006) (Figure 1). A cyclic pathway that starts from nicotinic acid mononucleotide performs de novo and salvage production of NAD, supplying nicotinic acid as an intermediate (Katoh and Hashimoto 2004). In contrast to the early steps that form the rings, little is known about the late steps responsible for ring coupling. Two orphan reductases, A622 of the PIP family (named after its founding members, pinoresinollariciresinol reductase, isoflavone reductase, and phenylcoumaran benzylic ether reductase) and berberine bridge enzyme-like protein (BBL) (belonging to a protein family including berberine bridge enzymes, carbohydrate oxidases, cannabinoid synthases, and 6-hydroxynicotine oxidases), are postulated to be involved in the late steps, but details of the reactions that they catalyze have yet to be defined (De Boer et al. 2009; Kajikawa et al. 2009; Kajikawa et al. 2011).

Once formed, nicotine moves dynamically through biological membranes via tonoplast-localized multidrug and toxic compound extrusion transporters (MATEs), MATE1, MATE2, and jasmonate-inducible alkaloid transporter 1 (JAT1) (Morita et al. 2009; Shoji et al. 2009), and plasma membrane-localized purine permease family member nicotine uptake permease 1 (NUP1) (Hildreth et al. 2011). There are likely to be 
further, unknown transporters for nicotine and pathway intermediates.

\section{ERF transcription factor genes cluster at the nicotine-controlling NICOTINE2 locus}

Mutants displaying altered nicotine levels are valuable resources for biosynthesis studies. A naturally occurring low-nicotine tobacco mutant was discovered in Europe in the early 1930s, and the trait was introduced through repeated introgressions into commercial tobacco cultivars to meet a demand for low-nicotine cigarettes. Through the breeding process, the genetic basis of this mutant became clear: mutant alleles at two distinct loci, NICOTINE1 and NICOTINE2 (NIC1 and NIC2, originally called the $A$ and $B$ loci), are responsible for the low-nicotine trait. Only a slight reduction in nicotine level is observed in either single mutant, nic1 having a stronger effect than nic2 (Legg and Collins 1971). Biochemical and molecular characterization of the lownicotine mutant revealed that multiple biosynthesis steps, rather than a single step, are blocked or markedly suppressed in the mutant, implying regulatory roles for the NIC loci (Saunders and Bush 1979; Shoji and Hashimoto 2013). Indeed, screening for genes suppressed in the mutant by three molecular approaches, cDNA subtraction (Hibi et al. 1994), differential display (Kidd et al. 2006; Shoji et al. 2009), and cDNA microarray (Kajikawa et al. 2011; Katoh et al. 2007; Shoji et al. 2010), led to the identification of nearly all of the known metabolic and transport genes involved in the nicotine pathway: ODC, PMT, MPO, AO, QS, QPT, A622, BBL, MATE1, MATE2, and NUP1.

Until recently, the molecular identities of the NIC genes had been long-standing questions. We found some clues to their identity through cDNA microarray analysis in a further search for genes suppressed in the low-nicotine mutant (Shoji et al. 2010). In addition to a series of structural genes of the biosynthetic pathway, the ERF189 gene, which encodes a transcription factor of the APETALA2 (AP2)/ethylene response factor (ERF) superfamily (Nakano et al. 2006), was found to be severely suppressed in the nic1nic2 mutant. In the tobacco genome, ERF189 and its close homologs belong to group IXa of the AP2/ERF superfamily (Rushton et al. 2008), members of which can be further divided into clade 1 and the ERF189-containing clade 2 (Figure 2). Characterization of these ERFs revealed that at least seven members of clade 2, including ERF189, are deleted altogether in the nic2 mutant (Figure 2); PCR did not detect the genes in the mutant genome and no expression of ERF189 was detected by RT-PCR in the nic2 mutant, while specific detection of the others is equivocal. Furthermore, since genetic analysis to examine linkage between the mutant allele and each gene deletion indicated a clustering of the deleted ERF genes

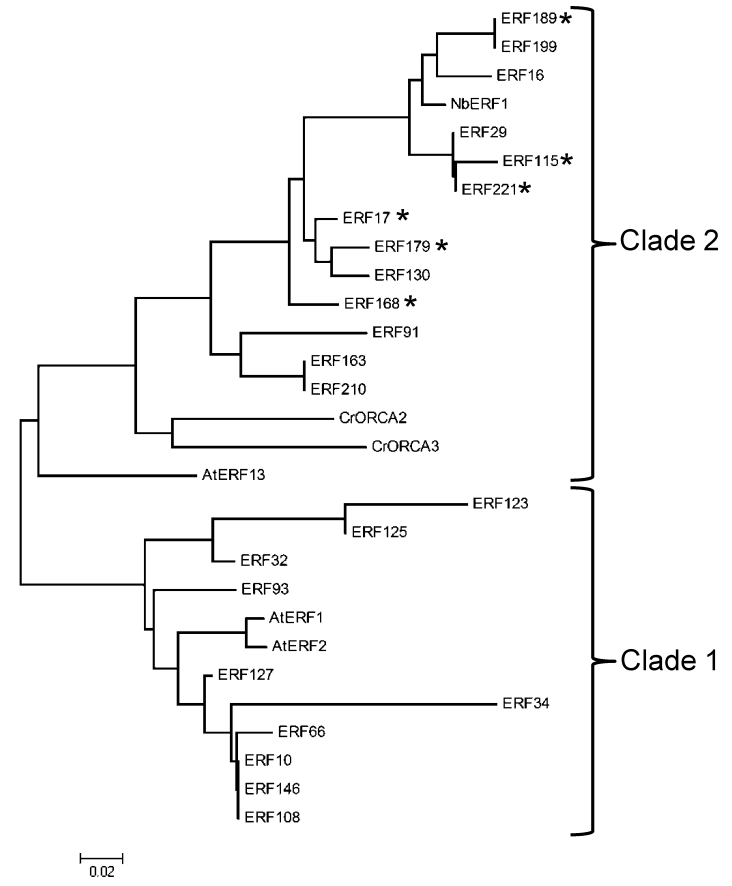

Figure 2. Phylogenetic relationship of group IXa ERF proteins. Based on the alignment of conserved DNA-binding AP2/ERF domain sequences, a phylogenetic tree was constructed by the neighbor-joining algorithm using MEGA4 (Tamura et al. 2007). The scale bar indicates the number of amino acid substitutions per site. Six of the seven ERFs whose genes are deleted in the nic2 mutant are indicated by asterisks. Although the gene encoding ERF104 is also deleted in nic2, ERF104 is not included in this tree because a stop codon in the AP2/ERF domain suggests that ERF104 is a pseudo-gene. AtERF1 (At4g17500), AtERF2 (At5g47220), and AtERF13 (At2g44840) are from Arabidopsis thaliana, CrORCA2 (AJ238740) and CrORCA3 (EU072424) are from Catharanthus roseus, NbERF1 (GQ859157) is from N. benthamiana, and others are from tobacco. Sequences of tobacco ERFs are available at TOBFAC (Rushton et al. 2008) under the same names.

at the NIC2 locus, the molecular basis of the mutation is presumably the extensive chromosomal deletion of these so-called NIC2-locus ERFs. Genomic sequencing of the region will determine whether this assumption is correct. Apart from the seven deleted genes, the existence in the genome and possibly the expression of the other group IXa members are not affected by the nic2 mutation, and so far no abnormality of any group IXa ERF tested has been found in the nic1 mutant at either the genomic or the transcript level (Shoji et al. 2010, and our unpublished data). The ancestral origin of the ERF genes clearly explains why only a fraction of the clade 2 ERFs are missing in nic2. NIC2-locus ERF genes may be derived from $N$. tomentosiformis, one of the two diploid ancestors of allotetraploid N. tabacum (Clarkson et al. 2005), and a hypothetical equivalent of the NIC2 locus from the other diploid ancestor, N. sylvestris, may contain the clade 2 ERF genes that are unchanged in nic2. The relatively mild phenotype of the nic 2 mutant may be attributed to the existence of such $N$. sylvestris-derived, functionally redundant ERF genes. 
NIC2-locus ERFs and closely related factors have also been proposed to be nicotine regulators in other studies based on a high-throughput trans-activation assay of the $P M T$ promoter using a transient expression system in tobacco protoplasts (De Sutter et al. 2005) and a functional screening using virus-induced gene silencing in N. benthamiana (Todd et al. 2010).

\section{A regulon for nicotine biosynthesis}

NIC2-locus ERFs directly up-regulate structural genes of the nicotine pathway, forming a nicotine biosynthesis regulon with their downstream target genes (Shoji et al. 2010; Shoji and Hashimoto 2011b; Shoji and Hashimoto 2012a). Over-expression of a certain member of NIC2locus ERF genes increased alkaloid level dramatically in the low-nicotine nic1nic2 mutant and several-fold in wild-type tobacco, which accumulates nicotine to a high level even without the over-expression (De Boer et al. 2011; Shoji et al. 2010). Conversely, loss-of-function of NIC2-locus ERFs, achieved by collective down-regulation of the genes either by RNA interference or by expression of the ERFs under the control of a dominant-repressive motif, drastically decreased the alkaloid content (Shoji et al. 2010). Using the glucocorticoid receptor fusion system, we observed activation of the nicotine pathway genes in response to steroid-induced activation of NIC2locus ERFs even in the presence of a protein synthesis inhibitor, suggesting that the structural genes of the pathway are activated directly by the ERF transcription factors (Shoji et al. 2010).

All NIC-controlled nicotine biosynthesis genes examined to date are immediate targets of NIC2-locus ERFs. At least eight GCC-like boxes recognized in vitro by NIC2-locus ERFs are present in the examined NICcontrolled promoter regions, and their consensus sequence, 5'-A/CGCA/CNNCCA/T-3' (Shoji and Hashimoto 2012a), is similar but not identical to the sequence of the canonical GCC box, 5' -AGCCGCC-3', a typical binding sequence for ERF factors, indicating that the NIC2-locus ERFs have a unique binding preference.

Based on the distribution of GCC-like boxes recognized by NIC2-locus ERFs in the NIC-controlled promoters, we could distinguish two QPT genes, QPT1 and $Q P T 2$, that encode an enzyme involved in the formation of the pyridine ring that is utilized for both NAD and alkaloid synthesis (Shoji and Hashimoto 2011b). Only the QPT2 promoter bears GCC-like boxes; in tobacco, three such boxes are in the proximal promoter region of $Q P T 2$, whereas none occur in that of $Q P T 1$. Accordingly, QPT2 is controlled by NIC2 locusERFs and is expressed in concert with other nicotine biosynthesis genes, while QPT1 expression is nearly constant in all tissues examined (Ryan et al. 2012; Shoji and Hashimoto 2011b). We infer that the QPT2 gene acquired the GCC-like boxes in its promoter to become involved in the nicotine biosynthesis regulon under the control of NIC2-locus ERFs, and that QPT2 supplies the pyridine ring that is increasingly demanded for nicotine synthesis, whereas the original role of sustaining metabolic flow for NAD synthesis is fulfilled by continuous QPT1 expression.

\section{Jasmonate signaling}

Chemical defense based on nicotine has been well studied in ecological terms (Baldwin 1998; Steppuhn et al. 2004). In addition to a basal level of production, nicotine synthesis readily increases in response to insect attack or wounding through signaling mediated by jasmonates (JAs) (Baldwin 1989; Baldwin et al. 1996). Damage-induced JA elevation in the leaf initiates the signaling, and movement of JA through the phloem ensures the systemic spread of the signal (Baldwin et al. 1994). Yet JA's role as systemically transmitted signal is still controversial and other possibilities cannot be excluded. In Nicotiana roots and cultured cells, JAs coordinately up-regulate the nicotine metabolic and transport genes $O D C, P M T, M P O, A O, Q S, Q P T, A 622$, $B B L, M A T E 1, M A T E 2, J A T 1$, and NUP1, all of which except JAT1 are also under the control of NIC genes (see above) (Shoji and Hashimoto 2011a; Shoji and Hashimoto 2013).

Molecular genetic studies using Arabidopsis have unveiled the basic framework of JA signaling from perception to gene activation (Browse 2009; Chung et al. 2009). A bioactive form of JA, JA-Ile, is perceived by a co-receptor complex of coronatine insensitive1 (COI1), an F-box component of an SCF-type E3-ubiquitin ligase complex (SCF ${ }^{\mathrm{COI} 1}$ ), and JA ZIM-domain (JAZ) proteins, triggering ubiquitination of JAZs by the ligase activity and their subsequent removal by $26 \mathrm{~S}$ proteasomemediated degradation (Chini et al. 2007; Thines et al. 2007). JAZ sequences are not highly conserved except for the Jas and TIFY motifs, which are important for proteinprotein interactions: homo- and heterodimer formation of JAZs depends on interactions involving the TIFY motif, whereas the Jas motif is at the interface for JAZCOI1 and JAZ-MYC2 (see below) interactions (Chini et al. 2009; Melotto et al. 2008). JAZs interact with various transcription factors and thereby connect them through an adaptor protein, novel interactor of JAZ (NINJA), to a Groucho/Tup1-type co-repressor, which suppresses transcription of nearby targeted genes, possibly through chromosomal remodeling (Pauwels et al. 2010). The transcription factors are liberated from the repressor complex after JA-dependent removal of JAZs, and regulate downstream genes both positively and negatively (Dombrecht et al. 2007). A list of the transcription factors targeted by JAZs includes the basic helix-loop-helix (bHLH) family member MYC2 and the related MYC3 and MYC4; the MYB family members MYB21 and 
- JA
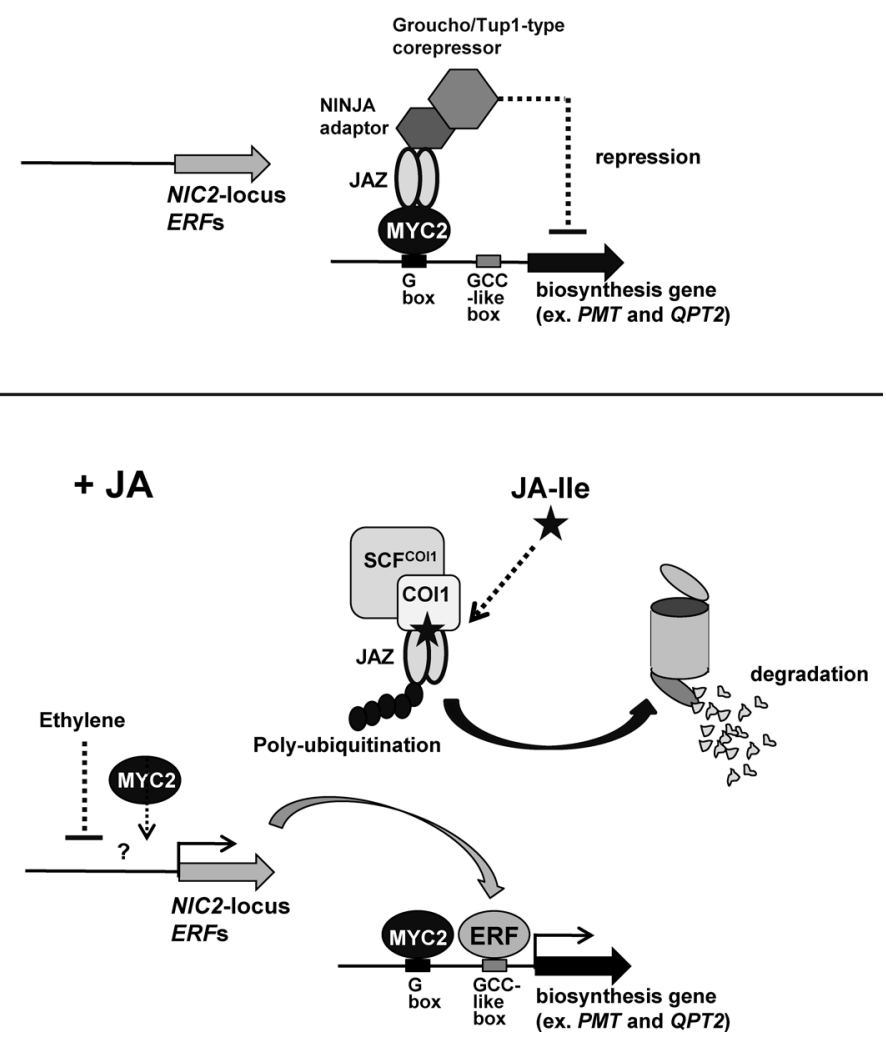

Figure 3. Model of JA-mediated regulation of nicotine biosynthesis. In the absence of JA signal input, JAZs interact with MYC2 and recruit a Groucho/Tup1-type co-repressor, which suppresses genes targeted by MYC2, through an adaptor protein NINJA. After JA-Ile is recognized by a co-receptor complex of COI1 and JAZ, JAZs are ubiquitinated by an SCF complex containing COI1 and then degraded by the 26S proteasome. The removal of JAZs allows downstream MYC2 to activate the genes for nicotine biosynthesis, either directly by binding to the corresponding promoters at the G box or indirectly by activating NIC2-locus ERFs. NIC2-locus ERF transcription factors up-regulate all metabolic and transport genes of nicotine pathway by binding at GCC-like boxes in their promoters. Ethylene signaling may suppress the transcription of NIC2-locus ERFs to negatively regulate the JA response of nicotine biosynthesis.

MYB24, which are required for stamen development; and complex-forming bHLH and R2R3-MYB family factors that mediate anthocyanin accumulation and trichome initiation (Chini et al. 2007; Fernandez-Calvo et al. 2011; Qi et al. 2011; Song et al. 2011; Thines et al. 2007).

The basic components in JA signaling are conserved evolutionarily and Nicotiana COI1, JAZs and MYC2 are all required for JA-induced nicotine formation (see below for MYC2) (Figure 3). Suppression of Nicotiana COI1 effectively impaired JA- and wound-induced nicotine formation along with other JA responses (Paschold et al. 2007; Shoji et al. 2008). Like their Arabidopsis counterparts, Nicotiana JAZ transcripts and proteins are induced and degraded, respectively, in response to JA (Oh et al. 2012; Shoji et al. 2008). Expression of truncated forms of JAZs lacking the C-terminal Jas motif, which may act in a dominant-negative manner by forming non-functional JAZ dimers, also clearly inhibited nicotine induction by JA (Shoji et al. 2008). Interestingly, RNA interference-mediated knockdown of a single JAZ member in N. attenuata, JAZh, significantly reduces nicotine levels, but enhances other JA-dependent responses (Oh et al. 2012). These latter observations, which seem contradictory at first glance, implicate mutual cross-regulation of JAZs, where JAZh may negatively regulate other $J A Z s$ that act as repressors of the nicotine response.

\section{Transcriptional regulators for the jasmonate response}

JA positively regulates nicotine pathway genes at the transcriptional level (Shoji et al. 2000a). As revealed for $P M T$ and QPT2 genes, JA-mediated induction of these genes depends on two distinct cis-elements in their proximal promoter regions, the G box and GCC-like box (Oki and Hashimoto 2004; Xu and Timko 2004), which are recognized by MYC2 and NIC2-locus ERFs, respectively (De Boer et al. 2011; Shoji et al. 2010; Shoji and Hashimoto 2011b; Shoji and Hashimoto 2011c; Todd et al. 2010; Zhang et al. 2012) (Figure 3). These transcription factors are also induced at the transcript level by JA and cooperatively activate their target promoters when expressed transiently in tobacco cells. Suppression of the transcription factor genes decreases 
both alkaloid level and the expression of genes involved in nicotine biosynthesis. While MYC2 is directly targeted by JAZ repressors and plays wide-ranging roles in JA signaling unrestricted to nicotine regulation, NIC2-locus ERFs are more specialized to regulating the nicotine pathway and do not interact with tested JAZs in the yeast two-hybrid assay (De Boer et al. 2011; Shoji and Hashimoto 2011c).

Little is yet known about how NIC2-locus ERFs are regulated. Some NIC2-locus ERF transcripts are immediately induced by JA, while others are gradually induced at later time points (Shoji et al. 2010). Ethylene signaling may integrate with JA signaling in the transcriptional regulation of NIC2-locus ERFs (Figure 3 ), since JA-mediated induction of the ERF transcripts along with those of downstream structural genes is suppressed by ethylene precursor treatment, even though these suppressive effects are incomplete and only apparent after prolonged treatment (Shoji et al. 2000b; 2010). When $M Y C 2$ was silenced by RNA interference in tobacco hairy roots, all NIC2-locus ERFs and thus their downstream structural genes were down-regulated. In contrast, MYC2 expression level was not altered either in nic mutants or in transgenic tobacco hairy roots in which a dominant-repressive form of a NIC2-locus ERF was over-expressed. These results suggest that MYC2 regulates NIC2-locus ERF genes, but not vice versa (Shoji and Hashimoto 2011c) (Figure 3). Further studies are necessary to address how individual NIC2-locus $E R F$ genes are regulated by MYC2, either directly or indirectly, and whether they are regulated at levels other than transcription.

\section{Regulatory function of proteins related to tobacco NIC2-locus ERFs}

Tobacco NIC2-locus ERFs are related to Catharanthus roseus ORCA2 and ORCA3 (Figure 2), which are known to regulate the JA response of terpenoid indole alkaloid biosynthesis in this species (van der Fits and Memelink 2000). The GCC-like boxes to which tobacco NIC2-locus ERFs bind can also be recognized by ORCA3 (Shoji and Hashimoto 2012a). ORCA3 is responsive to JA (van der Fits and Memelink 2000), and C. roseus MYC2 directly activates ORCA3 expression by recognizing a $\mathrm{G}$ box in its promoter (Zhang et al. 2011). These results indicate functional similarities between tobacco NIC2-locus ERFs and C. roseus ORCAs, in terms of their DNA-binding properties and of how they are regulated by JA and MYC2, and imply that functionally similar ERFs can be recruited to regulate JA-inducible metabolism in at least two distinct plant lineages, tobacco (Solanaceae) and periwinkle (Apocynaceae) (De Geyter et al. 2012; Shoji and Hashimoto 2012b).

Arabidopsis has three ERFs of group IXa, AtERF1, AtERF2, and AtERF13 (Figure 2), all of which are inducible by JA. AtERF1 and AtERF2 included in clade 1 are well-known founding members of AP2/ERF superfamily and can recognize a canonical GCC box $\left(5^{\prime}\right.$-AGCCGCC-3') found in various defensive genes (Fujimoto et al. 2000). AtERF13 that belongs to clade 2 including tobacco NIC2-locus ERFs and periwinkle ORCAs, was reported to be involved in abiotic tolerance (Lee et al. 2010). These facts suggest wider defensive roles of group IXa ERFs that are not restricted to JA-inducible alkaloid regulation.

\section{Perspectives}

We hope that the knowledge on nicotine regulation could be applicable to genetic engineering of other phytochemicals. Reflecting the defensive functions of natural products, elicitors that induce JA production and JA itself have been widely used to improve the productivity of useful phytochemicals in plant cells and tissue cultures (Blechert et al. 1995; Gundlach et al. 1992; Yukimune et al. 1996). It would be interesting to reveal whether the same kinds of ERFs as those described above are recruited for JA-dependent regulation of valuable phytochemicals. If they are, engineering aimed at transcription factors (Grotewold 2008) may give us promising ways to dynamically improve metabolic flow in selected pathways.

\section{Acknowledgements}

We thank Dr. Yasuyuki Yamada, Professor Emeritus of Nara Institute of Science and Technology (NAIST), for his continuous support and encouragement. We are grateful to Dr. Ian Smith (NAIST) for critical reading of the manuscript. This research was supported in part by a grant from the Japan Society for the Promotion of Science to T.S. (Grand-in-Aid for Scientific Research (C) No. 23570055)

\section{Addendum}

After the submission of this manuscript, Shoji et al. (2013) reported similar but divergent DNA-binding specificities of NIC2-locus ERFs and related transcription factors, such as ORCA3, AtERF1, and AtERF13. In this study, amino acid residues in the DNAbinding domain critical for such divergence were also determined.

\section{References}

Baldwin IT (1989) Mechanism of damage-induced alkaloid production in wild tobacco. J Chem Ecol 15: 1661-1680

Baldwin IT (1998) Jasmonate-induced responses are costly but benefit plants under attack in native populations. Proc Natl Acad Sci USA 95: 8113-8118

Baldwin IT, Schmelz EA, Ohnmeiss TE (1994) Wound-induced changes in root and shoot JA pools correlated with induced nicotine synthesis in Nicotiana sylvestris Spegazzini and Comes. JChem Ecol 20: 2139-2157

Baldwin IT, Schmelz EA, Zhang ZP (1996) Effects of octadecanoid metabolites and inhibitors on induced nicotine accumulation in Nicotiana sylvestris. J Chem Ecol 22: 61-74 
Blechert S, Brodschelm W, Hölder S, Kammerer L, Kutchan TM, Mueller MJ, Xia ZQ, Zenk MH (1995) The octadecanoic pathway: signal molecules for the regulation of secondary pathways. Proc Natl Acad Sci USA 92: 4099-4105

Browse J (2009) Jasmonate passes muster: a receptor and targets for the defense hormone. Annu Rev Plant Biol 60: 183-205

Chini A, Fonseca S, Fernández G, Adie B, Chico JM, Lorenzo O, Garcia-Casado G, López-Vidriero L, Lozano FM, Ponce MR, et al. (2007) The JAZ family of repressors is the missing link in jasmonate signalling. Nature 448: 666-671

Chini A, Fonseca S, Chico JM, Fernández-Calvo P, Solano R (2009) The ZIM domain mediate homo- and heteromeric interactions between Arabidopsis JAZ proteins. Plant J 59: 77-78

Chung HS, Niu Y, Browse J, Howe GA (2009) Top hits in contemporary JAZ: an update on jasmonate signaling. Phytochemistry 70: 1547-1559

Clarkson JJ, Lim KY, Kovarik A, Chase MW, Knapp S, Leitch AR (2005) Long-term genome diploidization in allopolyploid Nicotiana section Repandae (Solanaceae). New Phytol 168: 241-252

De Boer KD, Lye JC, Aitken CD, Su AK, Hamill JD (2009) The A622 gene in Nicotiana glauca (tree tobacco): Evidence for a functional role in pyridine alkaloid synthesis. Plant Mol Biol 69: 299-312

De Boer KD, Tilleman S, Pauwels L, Bossche RV, De Sutter V, Vanderhaeghen R, Hilson P, Hamill JD, Goossens A (2011) APETALA2/ETHYLENE RESPONSE FACTOR and basic helix-loop-helix transcription factors cooperatively mediate jasmonate-elicited nicotine biosynthesis. Plant J 66: 1053-1065

De Geyter N, Gholami A, Goormachtig S, Goossens A (2012) Transcriptional machineries in jamonate-elicited plant secondary metabolism. Trends Plant Sci 17: 349-359

De Sutter V, Vanderhaeghen R, Tilleman S, Lammertyn F, Vanhoutte I, Karimi M, Inzé D, Goossens A, Hilson P (2005) Exploration of jasmonate signalling via automated and standardized transient expression assays in tobacco cells. Plant J 44: 1065-1076

Dombrecht B, Xue GP, Sprague SJ, Kirkegaard JA, Ross JJ, Reid JB, Fitt GP, Sewelam N, Schenk PM, Manners JM, et al. (2007) MYC2 differentially modulates diverse jasmonate-dependent functions in Arabidopsis. Plant Cell 19: 2225-2245

Facchini PJ (2001) Alkaloid biosynthesis in plants: biochemistry, cell biology, molecular regulation, and metabolic engineering applications. Annu Rev Plant Physiol Plant Mol Biol 52: 29-66

Fernández-Calvo P, Chini A, Fernández-Barbero G, Chico JM, Gimenez-Ibanez S, Geerinck J, Eeckhout D, Schweizer F, Godoy M, Franco-Zorrilla JM, et al. (2011) The Arabidopsis bHLH transcription factors MYC3 and MYC4 are targets of JAZ repressors and act additively with MYC2 in the activation of jasmonate responses. Plant Cell 23: 701-715

Fujimoto SY, Ohta M, Usui A, Shinshi H, Ohme-Takagi M (2000) Arabidopsis ethylene-responsive element binding factors act as transcriptional activators or repressors of GCC box-mediated gene expression. Plant Cell 12: 393-404

Goodman J (1993) Tobacco in History: The Cultures of Dependence. Routledge, Abingdon, UK

Grotewold E (2008) Transcription factors for predictive plant metabolic engineering: are we there yet? Curr Opin Plant Biol 19: 138-144

Gundlach H, Müller MJ, Kutchan TM, Zenk MH (1992) Jasmonic acid is a signal transducer in elicitor-induced plant cell cultutes. Proc Natl Acad USA 89: 2389-2393
Hashimoto T, Yamada Y (1994) Alkaloid biogenesis: Molecular aspects. Annu Rev Plant Physiol Plant Mol Biol 45: 257-285

Hashimoto T, Yamada Y (2003) New genes in alkaloid metabolism and transport. Curr Opin Biotechnol 14: 163-168

Hecht SS (2003) Tobacco carcinogens, their biomarkers and tobacco-induced cancers. Nat Rev Cancer 3: 733-744

Hibi N, Higashiguchi S, Hashimoto T, Yamada Y (1994) Gene expression in tobacco low-nicotine mutants. Plant Cell 6: 723-735

Hildreth SB, Gehman EA, Yang H, Lu RH, Harich KC, Yu S, Lin J, Sandoe JL, Okumoto S, Murphy AS, et al. (2011) Tobacco nicotine uptake permease (NUP1) affects alkaloid metabolism. Proc Natl Acad Sci USA 108: 18179-18184

Kajikawa M, Hirai N, Hashimoto T (2009) A PIP-family protein is required for biosynthesis of tobacco alkaloids. Plant Mol Biol 69: 287-298

Kajikawa M, Shoji T, Katoh A, Hashimoto T (2011) Vacuolelocalized berberine bridge enzyme-like proteins are required for a late step of nicotine biosynthesis in tobacco. Plant Physiol 155: 2010-2022

Katoh A, Hashimoto T (2004) Molecular biology of pyridine nucleotide and nicotine biosynthesis. Front Biosci 9: 1577-1586

Katoh A, Uenohara K, Akita M, Hashimoto T (2006) Early steps in the biosynthesis of NAD in Arabidopsis start with aspartate and occur in the plastid. Plant Physiol 141: 851-857

Katoh A, Shoji T, Hashimoto T (2007) Molecular cloning of $N$ methylputrescine oxidase from tobacco. Plant Cell Physiol 48: 550-554

Kidd SH, Melillo AA, Lu RH, Reed DG, Kuno N, Uchida K, Furuya M, Jelesko JG (2006) The A and B loci in tobacco regulate a network of stress response gene, few of which are associated with nicotine biosynthesis. Plant Mol Biol 60: 699-716

Lee SJ, Park JH, Lee MH, Yu J, Kim SY (2010) Isolation and functional characterization of CE1 binding proteins. BMC Plant Biol 10: 277

Leete E (1983) Biosynthesis and metabolism of the tobacco alkaloids. In: Pelletier SW (ed) Alkaloids: Chemical and Biological Perspectives. John Wiley \& Sons, New York, pp 85-152

Legg PD, Collins GB (1971) Inheritance of percent total alkaloids in Nicotiana tabacum L. II. Genetic effects of two loci in Burley $21 \times$ LA Burley 21 populations. Can J Genet Cytol 13: 287-291

Matsuda J, Okada S, Hashimoto T, Yamada Y (1991) Molecular cloning of hyoscyamine $6 \beta$-hydroxylase, a 2 -oxoglutaratedependent dioxygenase, from cultured roots of Hyoscyamus niger. J Biol Chem 266: 9460-9464

Melotto M, Mecey C, Niu Y, Chung HS, Karsir L, Yao J, Zeng W, Thines B, Staswick P, Browse J, et al. (2008) A critical role of two positively charged amino acids in the Jas motif of Arabidopsis JAZ proteins in mediating coronatine- and jasmonoyl isoleucinedependent interactions with the COI1 F-box protein. Plant J 55: 979-988

Morita M, Shitan N, Sawada K, Van Montagu MC, Inzé D, Rischer H, Goossens A, Oksman-Caldentey KM, Moriyama Y, Yazaki K (2009) Vacuolar transport of nicotine is mediated by a multidrug and toxic compound extrusion (MATE) transporter in Nicotiana tabacum. Proc Natl Acad Sci USA 106: 2447-2452

Nakano T, Suzuki K, Fujimura T, Shinshi T (2006) Genome-wide analysis of the ERF gene family in Arabidopsis and rice. Plant Physiol 140: 411-432

Oh Y, Baldwin IT, Galis I (2012) NaJAZh regulates a subset of defense responses against herbivores and spontaneous leaf necrosis in Nicotiana attenuata plants. Plant Physiol 159: 769-788 
Oki H, Hashimoto T (2004) Jasmonate-responsive regions in a Nicotiana sylvestris PMT gene involved in nicotine biosynthesis. Plant Biotechnol 21: 269-274

Paschold A, Halitschke R, Baldwin IT (2007) Co(i)-ordinating defenses: NaCOI1 mediates herbivore-induced resistance in Nicotiana attenuate and reveals the role of herbivore movement in avoiding defenses. Plant J 51: 79-91

Pauwels L, Barbero GF, Geerinck J, Tilleman S, Grunewald W, Pérez AC, Chico JM, Bossche RV, Sewell J, Gil E, et al. (2010) NINJA connects the co-repressor TOPLESS to jasmonate signalling. Nature 464: 788-791

Qi T, Song S, Ren Q, Wu D, Huang H, Chen Y, Fan M, Peng W, Ren C, Xie D (2011) The Jasmonate-ZIM-domain proteins interact with the WD-repeat/bHLH/MYB complexes to regulate jasmonate-mediated anthocyanin accumulation and trichome initiation in Arabidopsis thaliana. Plant Cell 23: 1795-1814

Rushton PJ, Bokowiec MT, Laudeman TW, Brannock JF, Chen X, Timko MP (2008) TOBFAC: the database of tobacco transcription factors. BMC Bioinformatics 9: 53

Ryan SM, Cane KA, De Boer KD, Sinclair SJ, Brimblecombe R, Hamill JD (2012) Structure and expression of the quinolinate phosphoribosyltransferase (QPT) gene family in Nicotiana. Plant Sci 188: 102-110

Saunders JP, Bush LP (1979) Nicotine biosynthetic enzyme activities in Nicotiana tabacum L. genotypes with different alkaloid levels. Plant Physiol 64: 236-240

Shoji T, Yamada Y, Hashimoto T (2000a) Jasmonate induction of putrescine $N$-methyltransferase genes in the root of Nicotiana sylvestris. Plant Cell Physiol 41: 831-839

Shoji T, Nakajima K, Hashimoto T (2000b) Ethylene suppresses jasmonate-induced gene expression in nicotine biosynthesis. Plant Cell Physiol 41: 1072-1076

Shoji T, Ogawa T, Hashimoto T (2008) Jasmonate-induced nicotine formation in tobacco is mediated by tobacco COI1 and JAZ genes. Plant Cell Physiol 49: 1003-1012

Shoji T, Inai K, Yazaki Y, Sato Y, Takase H, Shitan N, Yazaki K, Goto Y, Toyooka K, Matsuoka K, et al. (2009) Multidrug and toxic compound extrusion-type transporters implicated in vacuolar sequestration of nicotine in tobacco roots. Plant Physiol 149: 708-718

Shoji T, Kajikawa M, Hashimoto T (2010) Clustered transcription factor genes regulate nicotine biosynthesis in tobacco. Plant Cell 22: 3390-3409

Shoji T, Hashimoto T (2011a) Nicotine biosynthesis. In: Ashihara H, Crozier A, Komamine A (eds) Plant Metabolism and Biotechnology. John Wiley \& Sons, New York, pp 191-216

Shoji T, Hashimoto T (2011b) Recruitment of a duplicated primary metabolism gene into the nicotine biosynthesis regulon in tobacco. Plant J 67: 949-959

Shoji T, Hashimoto T (2011c) Tobacco MYC2 regulates jasmonateinducible nicotine biosynthesis genes directly and by way of the NIC2-locus ERF genes. Plant Cell Physiol 52: 1117-1130

Shoji T, Hashimoto T (2012a) DNA-binding and transcriptional activation properties of tobacco NIC2-locus ERF189 and related transcription factors. Plant Biotechnol 29: 35-42

Shoji T, Hashimoto T (2012b) Jasmonate-responsive transcription factors: new tools for metabolic engineering and gene discovery. In: Chandra S, Lata H, Varma A (eds) Biotechnology for Medicinal Pants: Micropropagation and Improvement. Springer, Heidelberg, pp 345-357

Shoji T, Hashimoto T (2013) Biosynthesis and regulation of tobacco alkalioid. In: Wallner F (ed) Herbaceous Plants: Cultivation Methods, Grazing and Enviromental impacts. Nova Science Publishers, New York, pp 37-67

Shoji T, Mishima M, Hashimoto T (2013) Divergent DNA-binding specificities of a group of ETYHLENE RESPONSE FACTOR transcription factors involved in plant defense. Plant Physiol 162: 977-990

Song S, Qi T, Huang H, Ren Q, Wu D, Chang C, Peng W, Liu Y, Peng J, Xie D (2011) The Jasmonate-ZIM domain proteins interact with the R2R3-MYB transcription factors MYB21 and MYB24 to affect jasmonate-regulated stamen development. Plant Cell 23: 1000-1013

Steppuhn A, Gase K, Krock B, Halitschke R, Baldwin IT (2004) Nicotine's defensive function in nature. PLoS Biol 2: 1074-1080 Tamura B, Dudley J, Nei M, Kumar S (2007) MEGA4: Molecular Evolutionary Genetics Analysis (MEGA) software version 4.0. Mol Biol Evol 24: 1596-1599

Thines B, Katsir L, Melotto M, Niu Y, Mandaokar A, Liu G, Nomura K, He SY, Howe GA, Browse J (2007) JAZ repressor proteins are targets of the $\mathrm{SCF}^{\mathrm{CO}}{ }^{\mathrm{N}}$ complex during jasmonate signalling. Nature 448: 661-665

Todd AT, Liu E, Polvi SL, Pammett RT, Page JE (2010) A functional genomics screen identifies diverse transcription factors that regulate alkaloid biosynthesis in Nicotiana benthamiana. Plant $J$ 62: 589-600

van der Fits L, Memelink J (2000) ORCA3, a jasmonate-responsive transcriptional regulator of plant primary and secondary metabolism. Science 289: 295-297

Xu B, Timko MP (2004) Methyl jasmonate induced expression of the tobacco putrescine $N$-methyltransferase genes requires both G-box and GCC-motif elements. Plant Mol Biol 55: 743-761

Yukimune Y, Tabata H, Higashi Y, Hara Y (1996) Methyl jasmonate-induced overproduction of paclitaxel and baccatin III in Taxus cell suspension cultures. Nat Biotechnol 14: 1129-1132

Zhang H, Hedhili S, Montiel G, Zhang Y, Chatel G, Pré M, Gantet P, Memelink J (2011) The basic helix-loop-helix transcription factor CrMYC2 controls the jasmonate-responsive expression of the ORCA gene that regulate alkaloid biosynthesis in Catharanthus roseus. Plant J 67: 61-71

Zhang HB, Bokowiec MT, Rushton PJ, Han S-C, Timko MP (2012) Tobacco transcription factors NtMYC2a and NtMYC2b form nuclear complexes with the NtJAZ1 repressor and regulate multiple jasmonate-inducible steps in nicotine biosynthesis. $\mathrm{Mol}$ Plant 5: 73-84

Ziegler J, Facchini PJ (2008) Alkaloid biosynthesis: metabolism and trafficking. Annu Rev Plant Biol 59: 735-769 